\title{
Copula-based Multivariate GARCH Model with Uncorrelated Dependent Errors*
}

\author{
Tae-Hwy Lee ${ }^{\dagger}$ \\ University of California, Riverside
}

\author{
Xiangdong Long ${ }^{\ddagger}$ \\ University of Cambridge
}

August 2005

\begin{abstract}
Multivariate GARCH (MGARCH) models are usually estimated under multivariate normality. In this paper, for non-elliptically distributed financial returns, we propose copulabased multivariate GARCH (C-MGARCH) model with uncorrelated dependent errors, which are generated through a linear combination of dependent random variables. The dependence structure is controlled by a copula function. Our new C-MGARCH model nests a conventional MGARCH model as a special case. We apply this idea to the three MGARCH models, namely, the dynamic conditional correlation (DCC) model of Engle (2002), the varying correlation (VC) model of Tse and Tsui (2002), and the BEKK model of Engle and Kroner (1995). Monte Carlo experiment is conducted to illustrate the performance of C-MGARCH vs MGARCH models. Empirical analysis with a pair of the U.S. equity indices and two pairs of the foreign exchange rates indicates that the C-MGARCH models outperform DCC, VC, and BEKK in terms of in-sample model selection criteria (likelihood, AIC, SIC) and out-of-sample multivariate density forecast.
\end{abstract}

Key Words: Copula, Density forecast, Non-elliptical distribution, Predictive likelihood, Uncorrelated dependent errors.

JEL Classification: C3, C5, G0.

\footnotetext{
*Long thanks for the UCR Chancellor Fellowship.

${ }^{\dagger}$ Corresponding author. Department of Economics, University of California, Riverside, CA 92521, U.S.A. Tel: +1 (951) 827-1509. Fax: +1 (951) 827-5685. Email: tae.lee@ucr.edu.

${ }^{\ddagger}$ Cambridge Endowment for Research in Finance, Judge Institute of Management, University of Cambridge, U.K. Email: dxlecon@gmail.com.
} 


\section{Introduction}

Modeling the conditional covariance matrix is in the core of financial econometrics, as it is crucial for the asset allocation, financial risk management, and derivatives pricing. The multivariate generalized autoregressive conditional heteroskedasticity (MGARCH) models in the literature include the BEKK model by Engle and Kroner (1995), the dynamic conditional correlation (DCC) model by Engel (2002), and the varying correlation (VC) model by Tse and Tsui (2002). However, these models have been estimated under the multivariate normality assumption, while this assumption has been rejected in much of the empirical findings Fama and French (1993), Richardson and Smith (1993), Longin and Solnik (2001), Mashal and Zeevi (2002), among many others.

We propose a simple new model named a Copula-based Multivariate GARCH model, or in short C-MGARCH model, which permits modeling conditional correlation and dependence separately and simultaneously for interested financial returns with non-elliptically distributed dependent errors. Our approach is based on a transformation, which removes the linear correlation from the dependent variables to form uncorrelated dependent errors. The dependence structure is controlled by a copula while the correlation is modeled by an MGARCH model. The C-MGARCH model can capture the dependence in the uncorrelated errors ignored by all existing MGARCH models. For every MGARCH model, the corresponding C-MGARCH model can be constructed.

The paper is organized as follows. Section 2 provides a brief review on MGARCH models. Section 3 introduces the new C-MGARCH model with uncorrelated dependent errors. Monte Carlo simulation in Section 4 illustrates how MGARCH and C-MGARCH perform under the non-elliptical distributions, and shows the likelihood gains of the C-MGARCH models. Section 5 conducts empirical analysis for comparison of existing MGARCH models with their corresponding C-MGARCH models in terms of in-sample model selection criteria and outof-sample density predictive ability. The C-MGARCH models outperform corresponding DCC, VC and BEKK models when they are applied to a pair of the U.S. equity indices (NASDAQ and Dow Jones) and two pairs of the foreign exchange rates (French Franc and Deutschemark, and Japanese Yen and Deutschemark). Section 6 concludes. Section 7 is Appendix on copulas. 


\section{MGARCH Models}

We begin with a brief review of three MGARCH models. Suppose a vector of the $k$ return series $\left\{\mathbf{r}_{t}\right\}_{t=1}^{T}$ with $\mathbb{E}\left(\mathbf{r}_{t} \mid \mathcal{F}_{t-1}\right) \equiv \boldsymbol{\mu}_{t}$ and $\mathbb{E}\left(\mathbf{r}_{t} \mathbf{r}_{t}^{\prime} \mid \mathcal{F}_{t-1}\right) \equiv \mathbf{H}_{t}$ where $\mathcal{F}_{t-1}$ is the information set ( $\sigma$-field) at time $t-1$. For simplicity, we assume the conditional mean $\boldsymbol{\mu}_{t}$ is zero. For $\mathbf{H}_{t}$, many specifications have been proposed.

Engle and Kroner (1995) propose the BEKK model

$$
\mathbf{H}_{t}=\mathbf{C} \mathbf{C}^{\prime}+\mathbf{A}\left(\mathbf{r}_{t-1} \mathbf{r}_{t-1}^{\prime}\right) \mathbf{A}^{\prime}+\mathbf{B H}_{t-1} \mathbf{B}^{\prime}
$$

With the scalar or diagonal specifications on $\mathbf{A}$ and $\mathbf{B}$, we obtain the scalar BEKK or the diagonal BEKK.

Instead of modeling $\mathbf{H}_{t}$ directly, conditional correlation models decompose $\mathbf{H}_{t}$ into $\mathbf{D}_{t} \mathbf{R}_{t} \mathbf{D}_{t}$, where $\mathbf{D}_{t}^{2} \equiv \operatorname{diag}\left(\mathbf{H}_{t}\right)$. As the conditional covariance matrix for $\varepsilon_{t} \equiv \mathbf{D}_{t}^{-1} \mathbf{r}_{t}$ is the conditional correlation matrix for $\mathbf{r}_{t}$, Engle (2002) considers modeling $\mathbf{Q}_{t}$, the covariance matrix of $\varepsilon_{t}$, via a variance-targeting scalar BEKK model:

$$
\mathbf{Q}_{t}=(1-a-b) \overline{\mathbf{Q}}+a\left(\varepsilon_{t-1} \varepsilon_{t-1}^{\prime}\right)+b \mathbf{Q}_{t-1},
$$

where $\overline{\mathbf{Q}}$ is the sample covariance matrix of $\hat{\boldsymbol{\varepsilon}}_{t}$. A transformation $\mathbf{R}_{t}=\operatorname{diag} \mathbf{Q}_{t}^{-1} \mathbf{Q}_{t} \operatorname{diag} \mathbf{Q}_{t}^{-1}$ makes the conditional correlation matrix for $\mathbf{r}_{t}$.

The VC model of Tse and Tsui (2002) uses the following specification

$$
\mathbf{R}_{t}=(1-a-b) \overline{\mathbf{R}}+a \tilde{\mathbf{R}}_{t-1}+b \mathbf{R}_{t-1}
$$

where $\overline{\mathbf{R}}$ is the positive definite unconditional correlation matrix with ones in diagonal, and $\tilde{\mathbf{R}}_{t}=\sum_{i=1}^{M} \varepsilon_{1, t-i} \varepsilon_{2, t-i} /\left(\sum_{i=1}^{M} \varepsilon_{1, t-i}^{2} \sum_{i=1}^{M} \varepsilon_{2, t-i}^{2}\right)^{1 / 2} \cdot 1$

\section{New Model: C-MGARCH}

In the vast existing MGARCH literature, the distribution for $\mathbf{r}_{t}$ is assumed to be a certain bivariate elliptical distribution (e.g., bivariate normal or Student $t$ ) with mean $\boldsymbol{\mu}_{t}(=\mathbf{0})$

\footnotetext{
${ }^{1}$ In Tse and Tsui (2002), a necessary condition to guarantee $\tilde{\mathbf{R}}_{t}$ positive definite is $M \geqslant k$. Another necessary condition for non-singularity of $\tilde{\mathbf{R}}_{t}$, which should be added, is that $M$ should be bigger than the maximum number of observations of consecutive zeros of $\varepsilon_{i, t}, i=1, \ldots, k$. In the empirical section, we set $M=5$, which is transaction days in one week.
} 
and conditional covariance $\mathbf{H}_{t}$. The standardized errors $\mathbf{e}_{t}=\mathbf{H}_{t}^{-1 / 2} \mathbf{r}_{t}$ would then have the same bivariate elliptical distribution with zero mean and identity covariance: $\mathbb{E}\left(\mathbf{e}_{t} \mid \mathcal{F}_{t-1}\right)=$ 0 and $\mathbb{E}\left(\mathbf{e}_{t} \mathbf{e}_{t}^{\prime} \mid \mathcal{F}_{t-1}\right)=\mathbf{I}$. However, Embrechts et al. (1999) point out some wide-spread misinterpretations of the correlation, e.g., that no-correlation does not imply independence and a positive correlation does not mean positive dependence. Here, the identity conditional covariance matrix of $\mathbf{e}_{t}$ itself does not imply independence except when $\mathbf{e}_{t}$ follows an elliptical distribution.

The key point of this paper is that we permit dependence among the elements of $\mathbf{e}_{t}$ even if they are uncorrelated as shown by $\mathbb{E}\left(\mathbf{e}_{t} \mathbf{e}_{t}^{\prime} \mid \mathcal{F}_{t-1}\right)=\mathbf{I}$. The C-MGARCH model specifies the dependence structure and the conditional correlation separately and simultaneously. The former is controlled by a copula function and the latter is modeled by an MGARCH model for $\mathbf{H}_{t}$. Before we introduce our new C-MGARCH model, we first briefly review the copula theory (with some more details in Appendix).

\subsection{Copula}

Although there are many univariate distributions used in econometrics, for multivariate distribution there are few competitive candidates besides multivariate normal distribution and multivariate Student's $t$ distribution. However, the multivariate normal distribution is not consistent with the well-known asymmetry and excess kurtosis in financial data although it is easy to use. In this paper, we use the recently popular copulas to construct uncorrelated dependent errors. The principle characteristic of a copula function is its ability to decompose the joint distribution into two parts: marginal distributions and dependence structure. Different dependence structures can combine the same marginal distributions into different joint distributions. Similarly, different marginal distributions under the same dependence structure can also lead to different joint distributions. We focus on the bivariate case, which however can be easily extended to multivariate cases.

Definition (Copula): A function $C:[0,1]^{2} \rightarrow[0,1]$ is a copula if it satisfies (i) $C(u, v)=0$ for $u=0$ or $v=0$; (ii) $\sum_{i=1}^{2} \sum_{j=1}^{2}(-1)^{i+j} C\left(u_{i}, v_{j}\right) \geq 0$ for all $\left(u_{i}, v_{j}\right)$ in $[0,1]^{2}$ with $u_{1}<u_{2}$ and $v_{1}<v_{2}$; and (iii) $C(u, 1)=u, C(1, v)=v$ for all $u, v$ in $[0,1]$.

The relationship between a copula and joint distribution function is illuminated by Sklar's (1959) theorem. 
Theorem (Sklar): Let $K$ be a joint distribution function with margins $F$ and $G$. Then there exists a copula $C$ such that for all $\eta_{1}, \eta_{2}$ in $R$,

$$
K\left(\eta_{1}, \eta_{2}\right)=C\left(F\left(\eta_{1}\right), G\left(\eta_{2}\right)\right)=C(u, v) .
$$

Conversely, if $C$ is a copula and $F$ and $G$ are distribution functions, then the function $K$ defined above is a joint distribution function with margins $F$ and $G$.

The density $c(\cdot, \cdot)$ associated with $C(\cdot, \cdot)$ is $c(u, v)=\frac{\partial^{2} C(u, v)}{\partial u \partial v}$ and its relationship with the marginal density functions, $f(\cdot)$ and $g(\cdot)$, and the joint density function $k(\cdot)$ is

$$
k\left(\eta_{1}, \eta_{2}\right)=c\left(F\left(\eta_{1}\right), G\left(\eta_{2}\right)\right) \times f\left(\eta_{1}\right) \times g\left(\eta_{2}\right),
$$

where $f\left(\eta_{1}\right)=\partial F\left(\eta_{1}\right) / \partial \eta_{1}, g\left(\eta_{2}\right)=\partial G\left(\eta_{2}\right) / \partial \eta_{2}$ and $k\left(\eta_{1}, \eta_{2}\right)=\frac{\partial^{2} K\left(\eta_{1}, \eta_{2}\right)}{\partial \eta_{1} \partial \eta_{2}}$. For independent copula $C(u, v)=u v, c(u, v)=1$. An important property of copula function is its invariance under the increasing and continuous transformation, such as log transformation.

The joint survival function $\bar{C}(u, v)$ is $\bar{C}(u, v)=\operatorname{Pr}(U>u, V>v)=1-u-v+C(u, v)$. The survival copula of $C(u, v)$ is $C_{S}(u, v)=u+v-1+C(1-u, 1-v)$. The joint survival function and the survival copula are related through $\bar{C}(u, v)=C_{S}(1-u, 1-v)$. The density of survival copula can be expressed through the density of original copula as $c_{S}(u, v)=$ $c(1-u, 1-v)$.

Upper tail dependence $\lambda_{U}$ and lower tail dependence $\lambda_{L}$ defined as

$$
\begin{aligned}
& \lambda_{U}=\lim _{u \uparrow 1} \operatorname{Pr}\left[\eta_{2}>G^{-1}(u) \mid \eta_{1}>F^{-1}(u)\right]=\lim _{u \uparrow 1} \frac{[1-2 u+C(u, u)]}{1-u}, \\
& \lambda_{L}=\lim _{u \downarrow 0} \operatorname{Pr}\left[\eta_{2} \leqslant G^{-1}(u) \mid \eta_{1} \leqslant F^{-1}(u)\right]=\lim _{u \downarrow 0} \frac{C(u, u)}{u}
\end{aligned}
$$

measure the dependence in extreme cases. The tail dependence of each copula is discussed in Appendix.

In this paper, we use the independent (I) copula, Gumbel (G) copula, Clayton (C) copula, Frank (F) copula, Gumbel survival (GS) copula, Clayton survival (CS) copula, and Joe-Clayton (JC) copula. Their functional forms and properties are discussed in Appendix. From (5), the log-likelihood function is:

$$
\begin{aligned}
\mathcal{L}(\boldsymbol{\theta}, \boldsymbol{\alpha}) & =\sum_{t=1}^{T} \ln \left[f\left(\eta_{1, t} ; \boldsymbol{\theta}_{1}\right) g\left(\eta_{2, t} ; \boldsymbol{\theta}_{2}\right)\right]+\sum_{t=1}^{T} \ln c\left[F\left(\eta_{1, t} ; \boldsymbol{\theta}_{1}\right), G\left(\eta_{2, t} ; \boldsymbol{\theta}_{2}\right) ; \boldsymbol{\alpha}\right] \\
& \equiv \mathcal{L}_{M}(\boldsymbol{\theta})+\mathcal{L}_{C}(\theta, \boldsymbol{\alpha}),
\end{aligned}
$$


where $T$ is the number of the observations, $\boldsymbol{\theta}=\left(\boldsymbol{\theta}_{1}^{\prime} \boldsymbol{\theta}_{2}^{\prime}\right)^{\prime}$ are the parameters in the marginal densities $f(\cdot)$ and $g(\cdot)$, and $\boldsymbol{\alpha}$ is the copula shape parameter(s). The likelihood $\mathcal{L}(\boldsymbol{\theta}, \boldsymbol{\alpha})$ is decomposed into two parts, the first term $\mathcal{L}_{M}(\boldsymbol{\theta})$ is related to the marginal distributions and the second term $\mathcal{L}_{C}(\boldsymbol{\theta}, \boldsymbol{\alpha})$ is related to the copula.

\subsection{Models using copula}

We note that the MGARCH models discussed in Section 2 can also be put in the copula framework with elliptical copulas (normal or $t$ ). For example, to estimate for $\mathbf{H}_{t}=\mathbf{D}_{t} \mathbf{R}_{t} \mathbf{D}_{t}$, the DCC model of Engle (2002) assumes the normal margins for elements of $\varepsilon_{t}=\mathbf{D}_{t}^{-1} \mathbf{r}_{t}=$ $\left(\varepsilon_{1, t} \varepsilon_{2, t}\right)^{\prime}$ and the normal copula for $u_{t}=\Phi\left(\varepsilon_{1, t} ; \boldsymbol{\theta}_{1}\right)$ and $v_{t}=\Phi\left(\varepsilon_{2, t} ; \boldsymbol{\theta}_{2}\right)$ (where $\Phi(\cdot)$ is the univariate normal CDF) with the copula shape parameter being the time-varying conditional correlation $\mathbf{R}_{t}$. This is to assume the bivariate normal distribution. Let $\boldsymbol{\theta}$ be parameters in $\mathbf{D}_{t}$, and $\boldsymbol{\alpha}$ in $\mathbf{R}_{t}$. The log-likelihood function for the DCC model has the form:

$$
\begin{aligned}
\mathcal{L}(\boldsymbol{\theta}, \boldsymbol{\alpha}) & =-\frac{1}{2} \sum_{t=1}^{T} 2 \ln (2 \pi)+\mathbf{r}_{t}^{\prime} \mathbf{H}_{t}^{-1} \mathbf{r}_{t}+\ln \left|\mathbf{H}_{t}\right| \\
& =-\frac{1}{2} \sum_{t=1}^{T}\left(2 \ln (2 \pi)+\mathbf{r}_{t}^{\prime} \mathbf{D}_{t}^{-2} \mathbf{r}_{t}+\ln \left|\mathbf{D}_{t}\right|^{2}\right)-\frac{1}{2} \sum_{t=1}^{T}\left(\ln \left|\mathbf{R}_{t}\right|+\boldsymbol{\varepsilon}_{t}^{\prime} \mathbf{R}_{t}^{-1} \varepsilon_{t}-\boldsymbol{\varepsilon}_{t}^{\prime} \boldsymbol{\varepsilon}_{t}\right)
\end{aligned}
$$

where the first part corresponds to the normal marginal log-likelihood $\mathcal{L}_{M}(\boldsymbol{\theta})$ and the second part corresponds to the normal copula log-likelihood $\mathcal{L}_{C}(\boldsymbol{\theta}, \boldsymbol{\alpha})=\sum_{t=1}^{T} \ln c_{t}\left(F_{t}\left(\varepsilon_{1, t} ; \boldsymbol{\theta}_{1}\right)\right.$, $\left.G_{t}\left(\varepsilon_{2, t} ; \boldsymbol{\theta}_{2}\right) ; \boldsymbol{\alpha}\right)$ in (6). See (14) in Appendix. The margins contain $\mathbf{D}_{t}$ and the copula contains $\mathbf{R}_{t}$.

To accommodate the deviations from bivariate normality in the financial data, there have been other related attempts in the literature that use copulas. However, all of these works focus on modeling the conditional dependence instead of the conditional correlation. For example, taking the empirical distribution functions (EDF) for the margins and a parametric function for the copula, Breymann et al. (2003) and Chen and Fan (2005) estimate $\mathbf{D}_{t}^{2} \equiv$ $\operatorname{diag}\left(\mathbf{H}_{t}\right)$ using univariate realized volatility estimated from high frequency data or using univariate GARCH models. In that framework, estimated are the univariate conditional variances $\mathbf{D}_{t}^{2}$ and the conditional dependence, but not the conditional correlation $\mathbf{R}_{t}$ nor the conditional covariance $\mathbf{H}_{t}$ (if non-elliptical copulas are used).

The aim of this paper is to model $\mathbf{H}_{t}$ for non-elliptically distributed financial returns 
using non-elliptical copulas. We now introduce such a model. The idea is to have the new C-MGARCH model inherited from the existing $\mathrm{MGARCH}$ models to model $\mathbf{H}_{t}$, at the same time it is also to capture the remaining dependence in the uncorrelated dependent standardized errors $\mathbf{e}_{t}=\mathbf{H}_{t}^{-1 / 2} \mathbf{r}_{t}$.

\subsection{Structure of C-MGARCH model}

The C-MGARCH model can be formulated as follows:

$$
\begin{aligned}
\boldsymbol{\eta}_{t} \mid \mathcal{F}_{t-1} & \sim C\left(F_{t}(\cdot), G_{t}(\cdot) ; \alpha_{t}\right) \\
\mathbf{e}_{t} & =\Sigma_{t}^{-1 / 2} \boldsymbol{\eta}_{t} \\
\mathbf{r}_{t} & =\mathbf{H}_{t}^{1 / 2} \mathbf{e}_{t}
\end{aligned}
$$

where $\mathbb{E}\left(\mathbf{e}_{t} \mid \mathcal{F}_{t-1}\right)=\mathbf{0}, \mathbb{E}\left(\mathbf{e}_{t} \mathbf{e}_{t}^{\prime} \mid \mathcal{F}_{t-1}\right)=\mathbf{I}, \mathbb{E}_{(}\left(\boldsymbol{\eta}_{t} \mid \mathcal{F}_{t-1}\right)=\mathbf{0}, \mathbb{E}\left(\boldsymbol{\eta}_{t} \boldsymbol{\eta}_{t}^{\prime} \mid \mathcal{F}_{t-1}\right)=\Sigma_{t}=\left(\sigma_{i j, t}\right)$, and $C(\cdot, \cdot)$ is the conditional copula function.

The conventional approach is to assume bivariate independent normality for $\mathbf{e}_{t}(C(u, v)=$ $u v$, i.e., $\left.\sigma_{12}=0\right)$, while our approach is to assume a dependent copula for $\boldsymbol{\eta}_{t}=\left(\eta_{1, t} \eta_{2, t}\right)^{\prime}$ keeping $\mathbf{e}_{t}$ uncorrelated $\left(C(u, v) \neq u v\right.$, i.e., $\left.\sigma_{12} \neq 0\right)$. The main contribution of our CMGARCH model is that it permits modeling the conditional correlation and dependence structure, separately and simultaneously.

As the Hoeffding's (1940) lemma shows, the covariance between $\eta_{1}$ and $\eta_{2}$ is a function of marginal distributions $F(\cdot)$ and $G(\cdot)$, and joint distribution $K(\cdot)$. See Lehmann (1966) and Shea (1983).

Hoeffding's Lemma: Let $\eta_{1}$ and $\eta_{2}$ be random variables with the marginal distributions $F$ and $G$ and the joint distribution $K$. If the first and second moments are finite, then

$$
\sigma_{12} \equiv \operatorname{Cov}\left(\eta_{1}, \eta_{2}\right)=\iint_{\mathbb{R}^{2}}\left[K\left(\eta_{1}, \eta_{2}\right)-F\left(\eta_{1}\right) G\left(\eta_{2}\right)\right] d \eta_{1} d \eta_{2}
$$

By Hoeffding's Lemma and Sklar's Theorem, the off-diagonal element $\sigma_{12, t}$ of the conditional covariance matrix $\Sigma_{t}$ between $\eta_{1, t}$ and $\eta_{2, t}$ at time $t$, can be expressed as

$$
\sigma_{12, t}\left(\alpha_{t}\right)=\iint_{\mathbb{R}^{2}}\left[C\left(F_{t}\left(\eta_{1, t}\right), G_{t}\left(\eta_{2, t}\right) ; \alpha_{t}\right)-F_{t}\left(\eta_{1, t}\right) G_{t}\left(\eta_{2, t}\right)\right] d \eta_{1} d \eta_{2} .
$$


For simplicity, we assume that the marginal distributions and the copula shape parameter are not time-varying: $F_{t}(\cdot) \equiv F(\cdot), G_{t}(\cdot) \equiv G(\cdot)$, and $\alpha_{t} \equiv \alpha$. This makes $\sigma_{12, t}\left(\alpha_{t}\right) \equiv \sigma_{12}(\alpha)$ and $\Sigma_{t}\left(\alpha_{t}\right) \equiv \Sigma(\alpha){ }^{2}$

The log-likelihood function for $\boldsymbol{\eta}_{t}$ is:

$$
\mathcal{L}_{\eta}\left(\boldsymbol{\eta}_{t}\right)=\sum_{t=1}^{T} \ln f\left(\eta_{1, t}\right)+\ln g\left(\eta_{2, t}\right)+\ln c\left(F\left(\eta_{1, t}\right), G\left(\eta_{2, t}\right) ; \alpha\right) .
$$

Because $\mathbf{r}_{t}=\mathbf{H}_{t}^{1 / 2} \Sigma^{-1 / 2} \boldsymbol{\eta}_{t}$, the log-likelihood function for $\mathbf{r}_{t}$ is:

$$
\begin{aligned}
\mathcal{L}_{r}\left(\mathbf{r}_{t}\right) & =\mathcal{L}_{\eta}\left(\Sigma^{1 / 2} \mathbf{H}_{t}^{-1 / 2} \mathbf{r}_{t}\right)+\sum_{t=1}^{T} \ln \left|\Sigma^{1 / 2} \mathbf{H}_{t}^{-1 / 2}\right| \\
& =\sum_{t=1}^{T} \ln f\left(\eta_{1, t}\right)+\ln g\left(\eta_{2, t}\right)+\ln c\left(F\left(\eta_{1, t}\right), G\left(\eta_{2, t}\right) ; \alpha\right)+\ln \left|\Sigma^{1 / 2} \mathbf{H}_{t}^{-1 / 2}\right|,
\end{aligned}
$$

where $\left|\Sigma^{1 / 2} \mathbf{H}_{t}^{-1 / 2}\right|$ is the Jacobian of the transformation from $\boldsymbol{\eta}_{t}$ to $\mathbf{r}_{t}$. We use the maximum likelihood method to estimate all unknown parameters in one step. Under some regularity conditions, the usual asymptotic properties of ML estimators may be obtained.

Remark 1: Because $\mathbf{e}_{t}=\Sigma_{t}^{-1 / 2} \boldsymbol{\eta}_{t}$, if $\Sigma_{t}^{-1 / 2}=\Sigma^{-1 / 2} \equiv\left(a_{i j}\right)$, then $e_{1, t}=a_{11} \eta_{1, t}+a_{12} \eta_{2, t}$ and $e_{2, t}=a_{12} \eta_{1, t}+a_{22} \eta_{2, t}$ would be linear combinations of two dependent random variables $\eta_{1, t}$ and $\eta_{2, t}$. Even if each of $\eta_{1, t}$ and $\eta_{2, t}$ has the margins of standard normal distribution, the marginal distributions of $e_{1, t}$ and $e_{2, t}$ are not normal distribution because $\eta_{1, t}$ and $\eta_{2, t}$ are not independent.

Remark 2: The C-MGARCH model nests all existing MGARCH models. When the copula for $\boldsymbol{\eta}_{t}$ is independent copula, $\Sigma$ is diagonal. In addition, if marginal distributions for $\boldsymbol{\eta}_{t}$ are standard normal, the C-MGARCH model degenerates to the corresponding MGARCH model with bivariate normal distribution for $\mathbf{r}_{t}$. The C-MGARCH model inherits the dynamics of $\mathbf{H}_{t}$ from existing MGARCH models. For every MGARCH model, we can construct the corresponding C-MGARCH models with uncorrelated dependent errors.

Remark 3: The C-MGARCH models permit modeling conditional correlation and dependence separately and simultaneously with non-elliptically distributed dependent errors, and remove correlation from dependence to form the uncorrelated dependent errors. The remaining dependence is then captured by a copula. To our knowledge, no previous models

\footnotetext{
${ }^{2} \alpha_{t}$ may be time-varying. For example, $\alpha_{t}=1+\exp \left(a+b \alpha_{t-1}+c u_{t-1}+d v_{t-1}\right)$ for Gumbel copula, will make $1 \leq \alpha_{t}<\infty$. We leave this for the future research.
} 
incorporate correlation and dependence separately and simultaneously. Instead, they focus only on the dependence by modeling shape parameter in copula, Kendall's $\tau$, or Spearman's $\varrho$. However, as pointed out by Embrechts et al. (1999), the knowledge on Kendall's $\tau$ or Spearman's $\varrho$ alone can not help to choose the Markowitz risk-minimizing portfolio. The power of copulas is partly weakened by their unavailability of conditional covariance matrix. Different from the existing financial applications of copula theory, which focus on dependence and ignore the correlation, our C-MGARCH models are consistent with the mainstream methodology in financial industry - the variance-covariance approach of optimizing portfolio.

\section{Monte Carlo}

We conduct the simulations on samples of moderate size $T=500$ for bivariate systems simulated from the C-MGARCH models (8) with normal margins, with each of DCC, VC, and BEKK models for $\mathbf{H}_{t}$, and with Archimedean copulas. We then estimate C-MGARCH models with and without restricting $\sigma_{12}=0$, while keeping models of $\mathbf{H}_{t}$ (DCC, VC, BEKK) and marginal density (normal) correctly specified. The parameter $\sigma_{12}$ measures the dependence in the uncorrelated dependent error $\mathbf{e}_{t}$. The likelihood-ratio between the two C-MGARCH models with and without restricting $\sigma_{12}=0$ measures the dependence beyond the correlation (the dependence hidden in the uncorrelated errors). We also examine how the MLE performs in estimating the C-MGARCH models, particularly on the copula shape parameter $\alpha$ and $\sigma_{12}(\alpha)$.

The simulation takes the following steps. (i) Generate $\boldsymbol{\eta}_{t}$ from normal margins and a copula via the following method discussed in Nelsen (1999): draw two independent uniform $[0,1]$ variables $u$ and $p$, and get $v$ via $v=c_{u}^{-1}(p ; \alpha)$ where $c_{u}(p ; \alpha)=\partial C(u, v ; \alpha) / \partial u$. Then $\boldsymbol{\eta}_{t} \equiv\left(\eta_{1, t} \eta_{2, t}\right)^{\prime}=\left(\Phi^{-1}(u) \Phi^{-1}(v)\right)^{\prime}$ are the desired pair. ${ }^{3}$ (ii) Compute $\Sigma_{t}(\alpha)$ from the Hoeffding formula. Standardize $\boldsymbol{\eta}_{t}$ to get $\mathbf{e}_{t} \equiv \Sigma_{t}^{-1 / 2} \boldsymbol{\eta}_{t}$. (iii) For the copula-based DCC, get $\mathbf{H}_{t}=\mathbf{D}_{t} \mathbf{R}_{t} \mathbf{D}_{t}$, where $\mathbf{D}_{t}^{2}=\operatorname{diag}\left(h_{i, t} h_{2, t}\right), h_{1, t}=0.01+0.05 r_{1, t-1}^{2}+0.94 h_{1, t-1}, h_{2, t}=$ $0.5+0.2 r_{1, t-1}^{2}+0.5 h_{2, t-1}, \mathbf{Q}_{t}=\mathbf{C C}^{\prime}+0.2\left(\varepsilon_{t-1} \varepsilon_{t-1}^{\prime}\right)+0.7 \mathbf{Q}_{t-1}, \mathbf{C} \equiv\left(c_{i j}\right), c_{11}=0.4, c_{12}=$ $0, c_{21}=0.3, c_{22}=0.9$, and $\mathbf{R}_{t}=\operatorname{diag} \mathbf{Q}_{t}^{-1} \mathbf{Q}_{t} \operatorname{diag} \mathbf{Q}_{t}^{-1}$. We modify DCC into one-step estimation framework: to guarantee the positive definite of $\mathbf{Q}_{t}$, the Cholesky factorization

\footnotetext{
${ }^{3}$ We use a Matlab function, COPULARND, written by Perkins and Lane (2003) at MathWorks, Inc.
} 
$\mathbf{C C}^{\prime}$ is applied. The processes of $h_{1, t}$ and $h_{2, t}$ are same as Engle's (2002) simulation design with high persistency for one and low persistency for the other. (iv) Finally, obtain $\mathbf{r}_{t} \equiv$ $\mathbf{H}_{t}^{-1 / 2} \mathbf{e}_{t}$. We replicate this data generation process 200 times. For the copula-based VC model, Step (iii) is replaced with $\mathbf{R}_{t}=(1-0.2-0.7) \overline{\mathbf{R}}+0.2 \tilde{\mathbf{R}}_{t-1}+0.7 \mathbf{R}_{t-1}$ and the offdiagonal element of $\overline{\mathbf{R}}$ equal to 0.4. For the copula-based BEKK model, Step (iii) is replaced with $\mathbf{H}_{t}=\mathbf{C C}^{\prime}+0.05 \mathbf{r}_{t-1} \mathbf{r}_{t-1}^{\prime}+0.91 \mathbf{H}_{t-1}$ with the same $\mathbf{C}$ as for the DCC.

Table 1 presents the results for MGARCH (denoted I-MGARCH with I for independent copula) and C-MGARCH models. The results are reported only with Frank copula, but those with Clayton copula and Gumbel copula are available upon request. The results are more or less the same. Presented are the Monte Carlo averages and the Monte Carlo standard deviations of the estimated shape parameter $\hat{\alpha}$, the corresponding $\hat{\sigma}_{12} \equiv \sigma_{12}(\hat{\alpha})$, and the loglikelihood $(\log \mathrm{L})$. The correctly specified C-MGARCH model always has higher log-likelihood than the MGARCH model with wrong density assumption. The difference between the loglikelihood of the MGARCH model with the misspecified bivariate normal errors and that of the C-MGARCH model increases as the copula shape parameter $\alpha$ increases. For instance, for $\alpha=1$ and $\sigma_{12}=0.1570$, the log-likelihoods of I-DCC and F-DCC are about the same, -1473.6 and -1473.8 , respectively. For $\alpha=9$ and $\sigma_{12}=0.7919$, the log-likelihoods of I-DCC and F-DCC are -1451.1 and -1423.4 , respectively. The difference increases from -0.2 to 27.7. It is evident that the C-MGARCH model captures the dependence not captured by $\mathbf{H}_{t}$. For VC family, as the Frank copula shape parameter $\alpha$ increases from 1 to 9 , the difference of the log-likelihoods of I-VC and F-VC increases from -0.4 to 28.7. For BEKK family, as $\alpha$ increases from 1 to 9 , the difference increases from -0.04 to 29.95. These differences in $\log \mathrm{L}$ are the dependence not captured by $\mathbf{H}_{t}$ but captured by $\Sigma$ of the C-MGARCH models.

\section{Empirical Analysis}

The objective of this section is to compare the C-MGARCH models with the conventional MGARCH models in terms of in-sample estimation and out-of-sample forecasting. To elucidate the effect of the distinct feature $\left(\sigma_{12}\right)$ of the C-MGARCH model, we adopt the same normal marginal distribution and the same functional form of $\mathbf{H}_{t}$ for all the models under comparison, so that the difference arises only from the copula density.

We examine a pair of NASDAQ composite and Dow Jones Industrial Average (Dow), 
and two pairs of foreign exchange (FX) rate series (in U.S. dollars) - French Franc (FF) and Deutschemark (DM), and Japanese Yen (JY) and Deutschemark. The return series are 100 times the log difference of the stock indices or exchange rates. The daily U.S. equity indices are obtained from finance.yahoo.com, and the daily spot FX series are from the Federal Reserve Statistical Release. The sample periods of the data, out-of-sample forecast validation periods, and the size of rolling window to train forecasting models are as follows:

\begin{tabular}{|l|l|l|}
\hline & \multicolumn{1}{|c|}{ In-sample estimation } & \multicolumn{1}{c|}{ Out-of-sample forecasting } \\
\hline NASDAQ-DOW & $\begin{array}{l}\text { Jan. 2, 1997 to Dec. 31, 2001 } \\
T=1257\end{array}$ & $\begin{array}{l}\text { Jan. 4, 2001 to Dec. 31, 2001 } \\
P=248 \text { with rolling window } R=1009\end{array}$ \\
\hline FF-DM & $\begin{array}{l}\text { Jan. 4, 1993 to Dec. 31, 1998 } \\
T=1509\end{array}$ & $\begin{array}{l}\text { Jan. 7, 1997 to Dec. 31, 1998 } \\
P=500 \text { with rolling window } R=1009\end{array}$ \\
\hline JY-DM & $\begin{array}{l}\text { Jan. 4, 1993 to Dec. 31, 1997 } \\
T=1257\end{array}$ & $\begin{array}{l}\text { Jan. 5, 1997 to Dec. 31, 1997 } \\
P=250 \text { with rolling window } R=1007\end{array}$ \\
\hline
\end{tabular}

\section{$5.1 \quad$ In-sample estimation}

For the in-sample comparison between our C-MGARCH models and MGARCH models, Table 2 presents three model selection criteria (likelihood, AIC, SIC). For the NASDAQDOW pair and for the FF-DM pair, Gumbel Survival copula or Joe-Clayton copula are selected. Joe-Clayton copula nests Clayton copula as a special case. Gumbel Survival copula, Joe-Clayton copula, and Clayton copula all have the asymmetric tail dependence with positive lower tail dependence $\left(\lambda_{L}>0\right)$ and zero upper tail dependence $\left(\lambda_{U}=0\right)$. This characteristic implies so called "crashing-together". For the JY-DM pair, the model selection criteria select Frank copula, which has symmetric tail dependence. The log-likelihood ratio of two models is the entropy gain in the sense of Vuong (1989). The superiority of Gumbel Survival copula and Joe-Clayton copula (for NASDAQ-DOW and FF-DM) and Frank copula (for JY-DM) over the independent copula (i.e., multivariate normal distribution) indicates that the conditional joint distributions of these three pairs of equity and FX return series are not bivariate normal.

Table 2 also presents the estimated shape parameters $\hat{\boldsymbol{\alpha}}$, their robust standard errors, and the corresponding $\hat{\sigma}_{12}$. For FF-DM, the estimated shape parameters for the Gumbel Survival copula and Joe-Clayton copula are very significant. For JY-DM, the estimated shape parameter of Frank copula is 8.44 in Frank-DCC, 8.38 in Frank-VC and 6.98 in Frank-BEKK, 
and all significantly positive, indicating the remaining dependence in the standardized uncorrelated errors $\mathbf{e}_{t}$ is strong, positive, and symmetric in both tails.

\subsection{Out-of-sample predictive ability}

Suppose there are $l+1$ models in a set of the competing density forecast models, possibly misspecified. We compare $l=6 \mathrm{C}$-MGARCH models with the benchmark MGARCH model. Let these models be indexed by $j(j=0,1, \ldots, l=6)$ with the $j$ th density forecast model denoted by $\psi_{t}^{j}\left(\mathbf{r}_{t} ; \hat{\boldsymbol{\theta}}_{R, t-1}^{j}\right)$. The benchmark model is indexed with $j=0$. If a bivariate density forecast model $\psi_{t}\left(\mathbf{r}_{t} ; \boldsymbol{\theta}_{0}\right)$ coincides with the true density $\varphi_{t}\left(\mathbf{r}_{t}\right)$ almost surely for some $\boldsymbol{\theta}_{0} \in \Theta$, then the one-step-ahead density forecast is said to be optimal because it dominates all other density forecasts for any loss functions (Diebold et al., 1998; Granger and Pesaran, 2000). As in practice it is rarely the case that we can find an optimal model, our task is to investigate which density forecast model approximates the true conditional density most closely. If a metric is defined to measure the distance of a given model to the truth, we then compare different models in terms of this distance.

Following Bao et al. (2005), we compare C-MGARCH models by comparing the conditional Kullback-Leibler information criterion (KLIC), $\mathbb{I}_{t}\left(\varphi: \psi^{j}, \boldsymbol{\theta}\right)=\mathbb{E}_{\varphi_{t}} \ln \left[\varphi_{t}\left(y_{t}\right) / \psi_{t}^{j}\left(y_{t} ; \boldsymbol{\theta}^{j}\right)\right]$, where the expectation is with respect to the true conditional density $\varphi_{t}\left(\cdot \mid \mathcal{F}_{t-1}\right)$. Following White (1994), we define the distance between a density model and the true density as the minimum KLIC, $\mathbb{I}_{t}\left(\varphi: \psi^{j}, \boldsymbol{\theta}_{t-1}^{* j}\right)=\mathbb{E}_{\varphi_{t}} \ln \left[\varphi_{t}\left(y_{t}\right) / \psi_{t}^{j}\left(y_{t} ; \boldsymbol{\theta}_{t-1}^{* j}\right)\right]$, where $\boldsymbol{\theta}_{t-1}^{* j}=$ $\arg \min \mathbb{I}_{t}\left(\varphi: \psi^{j}, \boldsymbol{\theta}^{j}\right)$ is the pseudo-true value of $\boldsymbol{\theta}^{j}$. To estimate $\boldsymbol{\theta}_{t-1}^{* j}$, we split the data into two parts - one for the estimation and the other for out-of-sample validation. At each period $t$ in the out-of-sample period $(t=R+1, \ldots, T)$, we use the previous $R$ rolling observations $\left\{y_{t-1}, \ldots, y_{t-R}\right\}_{t=R+1}^{T}$ to estimate the unknown parameter vector $\boldsymbol{\theta}_{t-1}^{* j}$ and denote the estimate as $\hat{\boldsymbol{\theta}}_{R, t-1}^{j} \cdot{ }^{4}$ Under some regularity conditions, we can consistently estimate $\boldsymbol{\theta}_{t-1}^{* j}$ by $\hat{\boldsymbol{\theta}}_{R, t-1}^{j}$ by maximizing $R^{-1} \sum_{s=t-R+1}^{t} \ln \psi_{s}^{j}\left(y_{s} ; \boldsymbol{\theta}^{j}\right)$. See White (1994) for the sets of conditions for the existence and consistency of $\hat{\boldsymbol{\theta}}_{R, t-1}^{j}$.

Using $\left\{\hat{\boldsymbol{\theta}}_{R, t-1}\right\}_{t=R+1}^{T}$, we can obtain the out-of-sample estimate of $\mathbb{E}_{t}\left(\varphi: \psi^{j}, \boldsymbol{\theta}_{t-1}^{* j}\right)$ by $\mathbb{I}_{R, n}(\varphi: \psi) \equiv P^{-1} \sum_{t=R+1}^{T} \ln \left[\varphi_{t}\left(y_{t}\right) / \psi_{t}^{j}\left(y_{t} ; \hat{\boldsymbol{\theta}}_{R, t-1}^{j}\right)\right]$, where $P \equiv T-R$ is the size of the outof-sample period. Since the KLIC takes a smaller value when a model is closer to the truth,

\footnotetext{
${ }^{4}$ Alternatively, $\boldsymbol{\theta}_{t-1}^{*}$ can be estimated based on the whole subsample $\left\{y_{t-1}, \ldots, y_{1}\right\}$ or a fixed sample $\left\{y_{R}, \ldots, y_{1}\right\}$.
} 
we can regard it as a loss function. Bao et al. (2005) note that the out-of-sample average of the KLIC-differential between the benchmark model 0 and model $j$ is then simply the $\log$ ratio of the predictive likelihoods

$$
\mathbb{I}_{R, n}\left(\varphi: \psi^{0}\right)-\mathbb{I}_{R, n}\left(\varphi: \psi^{j}\right)=\frac{1}{P} \sum_{t=R+1}^{T} \ln \left[\psi_{t}^{j}\left(y_{t} ; \hat{\boldsymbol{\theta}}_{R, t-1}^{j}\right) / \psi_{t}^{0}\left(y_{t} ; \hat{\boldsymbol{\theta}}_{R, t-1}^{0}\right)\right] .
$$

When we compare multiple $l$ C-MGARCH models using various copulas against a benchmark MGARCH model, the null hypothesis of interest is that no C-MGARCH model is better than the benchmark MGARCH. White (2000) proposes a test statistic and the bootstrap procedure to compute its $p$-value.

Table 3 reports the density forecast comparison in terms of the out-of-sample KLIC together with the reality check p-values. In general, the in-sample results in Table 2 and the out-of-sample results in Table 3 are consistent, in that the in-sample ranking across the C-MGARCH models is carried over to the out-of-sample predictive ranking of the models.

For NASDAQ-DOW and for FF-DM, the density forecast comparison confirms the nonnormality. For all three families (DCC, VC, BEKK), the independent copula yields the smallest (worst) predictive likelihood among all seven C-MGARCH models. The predictive superiority of C-MGARCH models based on Gumbel Survival copula or Joe-Clayton copula over the MGARCH model based on the independent copula (MGARCH under the bivariate normal distribution) is marginally significant for NASDAQ-DOW (with the reality check p-values 0.087, 0.095, 0.195, respectively for DCC, VC, BEKK families) and is strongly significant for FF-DM (with the reality check p-values 0.002, 0.000, 0.008 respectively for DCC, VC, BEKK families). For these two pairs of return series, Gumbel Survival or JoeClayton C-MGARCH is significantly better than the benchmark even after accounting for potential bias due to the specification search.

For JY-DM, the independent copula yields one of the worst predictive likelihood. The largest (best) predictive likelihood is obtained from the Frank C-MGARCH for all three families. The result for JY-DM is weaker than for FF-DM, as the reality check p-values are 0.184, 0.144, 0.181, respectively for DCC, VC, BEKK families. This result is consistent with the results of Chen and Fan (2005, Section 6.1), who find much stronger rejection of normality for FF-DM than for JY-DM. They also find much stronger tail dependence for FF-DM than for JY-DM. 


\section{Conclusions}

In this paper we propose a new MGARCH model, namely, the C-MGARCH model. The C-MGARCH model includes a conventional MGARCH model as a special case. The CMGARCH model is to exploit the fact that the uncorrelated errors are not necessarily independent. The C-MGARCH model permits modeling the conditional covariance for the non-elliptically distributed financial returns, and at the same time separately modeling the dependence structure beyond the conditional covariance. While we have considered here

only a bivariate system, the extension to a higher dimension is straightforward. We compare the C-MGARCH models with the corresponding MGARCH models using the three financial data sets - a pair of the U.S. equity indices and two pairs of the foreign exchange rates. The empirical results from the in-sample and out-of-sample analysis clearly demonstrate the advantages of the new model.

\section{Appendix}

We present here some details on copula functions for two widely used copula families elliptical copula family and Archimedean copula family. The former includes the Gaussian copula and the Student's $t$ copula. The later includes Gumbel copula, Clayton copula and Frank copula. We also discuss the survival copulas of Archimedean copulas and Joe-Clayton copula.

\subsection{Elliptical copulas}

Gaussian copula: Let $\mathbf{R}$ be the symmetric, positive definite correlation matrix and $\Phi_{\mathbf{R}}(\cdot, \cdot)$ be the standard bivariate normal distribution with correlation matrix $\mathbf{R}$. The density function of bivariate Gaussian copula is:

$$
c^{\text {Gaussian }}(u, v)=\frac{1}{|\mathbf{R}|^{1 / 2}} \exp \left(-\frac{1}{2} \boldsymbol{\eta}^{\prime}\left(\mathbf{R}^{-1}-\mathbf{I}\right) \boldsymbol{\eta}\right),
$$

where $\boldsymbol{\eta}=\left(\Phi^{-1}(u) \Phi^{-1}(v)\right)^{\prime}$ and $\Phi^{-1}(\cdot)$ is the inverse of the univariate normal CDF. The bivariate Gaussian copula is:

$$
C^{\text {Gaussian }}(u, v ; \mathbf{R})=\Phi_{\mathbf{R}}\left(\Phi^{-1}(u), \Phi^{-1}(v)\right)
$$


Hu (2003) shows the bivariate Gaussian copula can be approximated by Taylor expansion:

$$
C^{\text {Gaussian }}(u, v ; \rho) \approx u v+\rho \phi\left(\Phi^{-1}(u)\right) \phi\left(\Phi^{-1}(v)\right),
$$

where $\phi$ is the density function of univariate Gaussian distribution and $\rho$ is the correlation coefficient between $\eta_{1}$ and $\eta_{2}$. Both the upper tail dependence $\lambda_{U}$ and the lower tail dependence $\lambda_{L}$ are zero, reflecting the asymptotic tail independence of Gaussian copula.

Student's $t$ copula: Let $\omega_{c}$ be the degree of freedom, and $\mathbf{T}_{\mathbf{R}, \omega_{c}}(\cdot, \cdot)$ be the standard bivariate Student's $t$ distribution with degree of freedom $\omega_{c}$ and correlation matrix $\mathbf{R}$. The density function of bivariate Student's $t$ copula is:

$$
c^{\text {Student's } t}\left(u, v ; \mathbf{R}, \omega_{c}\right)=|\mathbf{R}|^{-\frac{1}{2}} \frac{\Gamma\left(\frac{\omega_{c}+2}{2}\right) \Gamma\left(\frac{\omega_{c}}{2}\right)}{\Gamma\left(\frac{\omega_{c}+1}{2}\right)^{2}} \frac{\left(1+\frac{\eta^{\prime} R^{-1} \eta}{\omega_{c}}\right)^{-\frac{\omega_{c}+2}{2}}}{\prod_{i=1}^{2}\left(1+\frac{\eta_{i}^{2}}{\omega_{c}}\right)^{-\frac{\omega_{c}+1}{2}}}
$$

where $\boldsymbol{\eta}=\left(t_{\omega_{c}}^{-1}(u), t_{\omega_{c}}^{-1}(v)\right)^{\prime}, u=t_{\omega_{1}}(x), v=t_{\omega_{2}}(y)$, and $t_{\omega_{i}}(\cdot)$ is the univariate Student's $t$ $\mathrm{CDF}$ with degree of freedom $\omega_{i}$. The bivariate Student's $t$ copula is

$$
C^{\text {Student's } t}\left(u, v ; \mathbf{R}, \omega_{c}\right)=\mathbf{T}_{\mathbf{R}, \omega_{c}}\left(t_{\omega_{c}}^{-1}(u), t_{\omega_{c}}^{-1}(v)\right) .
$$

The upper tail dependence $\lambda_{U}$ of Student's $t$ copula is $\lambda_{U}=2-2 t_{\omega_{c}+1}\left(\sqrt{\omega_{c}+1} \sqrt{1-\rho} / \sqrt{1+\rho}\right)$, where $\rho$ is the off-diagonal element of $\mathbf{R}$. Because of the symmetry property, the lower tail dependence $\lambda_{L}$ can be obtained easily.

\subsection{Archimedean copulas}

Archimedean copula can be expressed as

$$
C(u, v)=\varphi^{-1}(\varphi(u)+\varphi(v)),
$$

where $\varphi$ is a convex decreasing function, called generator. Different generator will induce different copula in the family of Archimedean copula. The Kendall's $\tau=1+4 \int_{0}^{1} \frac{\varphi(u)}{\varphi^{\prime}(u)} d u$.

Gumbel copula: The generator for Gumbel copula is $\varphi_{\alpha}(x)=(-\ln x)^{\alpha}$. For $1 \leq \alpha<\infty$ ( $\alpha=1$ for independence and $\alpha \rightarrow \infty$ for more dependence), the CDF and PDF for Gumbel copula are

$$
\begin{aligned}
C^{\text {Gumbel }}(u, v ; \alpha) & =\exp \left\{-\left[(-\ln u)^{\alpha}+(-\ln v)^{\alpha}\right]^{1 / \alpha}\right\} \\
c^{\text {Gumbel }}(u, v ; \alpha) & =\frac{C^{\text {Gumbel }}(u, v ; \alpha)(\ln u \ln v)^{\alpha-1}\left\{\left[(-\ln u)^{\alpha}+(-\ln v)^{\alpha}\right]^{1 / \alpha}+\alpha-1\right\}}{u v\left[(-\ln u)^{\alpha}+(-\ln v)^{\alpha}\right]^{2-1 / \alpha}} .
\end{aligned}
$$


The Kendall's $\tau$ for Gumbel copula is $\tau=1-\frac{1}{\alpha}$. This one-to-one mapping relationship between $\tau$ and $\alpha$ clearly shows the shape parameter $\alpha$ controlling the dependence structure. The dependence structure of Gumbel copula are asymmetric: $\lambda_{U}=2-2^{1 / \alpha}$ and $\lambda_{L}=0$.

The survival copula of Gumbel copula has mirror image to Gumbel copula. Its CDF and PDF are

$$
\begin{aligned}
C^{\mathrm{GS}}(u, v ; \alpha) & =u+v-1+\exp \left\{-\left[(-\ln (1-u))^{\alpha}+(-\ln (1-v))^{\alpha}\right]^{1 / \alpha}\right\}, \quad \alpha \in[1, \infty) \\
c^{\mathrm{GS}}(u, v ; \alpha) & =c^{\mathrm{Gumbel}}(1-u, 1-v ; \alpha) .
\end{aligned}
$$

The Kendall's $\tau$ for Gumbel survival copula is $\tau=1-\frac{1}{\alpha}$. Gumbel Survival copula has the positive lower tail dependence: $\lambda_{U}=0$ and $\lambda_{L}=2-2^{1 / \alpha}$.

Clayton copula: The generator for Clayton copula is $\varphi_{\alpha}(x)=\frac{x^{-\alpha}-1}{\alpha}$. For $\alpha \in$ $[-1, \infty) \backslash\{0\}$, the CDF and the PDF for Clayton copula are

$$
\begin{aligned}
C^{\text {Clayton }}(u, v ; \alpha) & =\left(u^{-\alpha}+v^{-\alpha}-1\right)^{-1 / \alpha} \\
c^{\text {Clayton }}(u, v ; \alpha) & =\frac{(1+\alpha)\left(u^{-\alpha}+v^{-\alpha}-1\right)^{-\frac{1}{\alpha}-2}}{(u v)^{\alpha+1}} .
\end{aligned}
$$

The Kendall's $\tau$ for Clayton copula is $\frac{\alpha}{\alpha+2}$. The upper tail dependence $\lambda_{U}=0$ and the lower tail dependence is $\lambda_{L}=2^{-1 / \alpha}$. Joe-Clayton copula nests Clayton copula as a special case. The CDF of Joe-Clayton copula is

$$
C^{\text {Joe-Clayton }}\left(u, v ; \alpha_{1}, \alpha_{2}\right)=1-\left(1-\left\{\left[1-(1-u)^{\alpha_{1}}\right]^{-\alpha_{2}}+\left[1-(1-v)^{\alpha_{1}}\right]^{-\alpha_{2}}-1\right\}^{-1 / \alpha_{2}}\right)^{1 / \alpha_{1}} .
$$

When $\alpha_{1}=1$, Joe-Clayton copula generates to Clayton copula.

Frank copula: The generator for Frank copula is $\varphi_{\alpha}(x)=-\ln \left(\frac{e^{-\alpha x}-1}{e^{-\alpha}-1}\right)$. For $\alpha \in \mathbb{R} \backslash\{0\}$, the CDF and PDF for Frank copula are

$$
\begin{aligned}
C^{\text {Frank }}(u, v ; \alpha) & =-\frac{1}{\alpha} \log \left[1-\frac{\left(1-e^{-\alpha u}\right)\left(1-e^{-\alpha v}\right)}{\left(1-e^{-\alpha}\right)}\right] \\
c^{\text {Frank }}(u, v ; \alpha) & =\frac{\alpha\left(1-e^{-\alpha}\right) e^{-\alpha(u+v)}}{\left[\left(1-e^{-\alpha}\right)-\left(1-e^{-\alpha u}\right)\left(1-e^{-\alpha v}\right)\right]^{2}} .
\end{aligned}
$$

The dependence structure described by Frank copula is symmetric: $\alpha>0$ for positive dependence, $\alpha \rightarrow 0$ for independence, and $\alpha<0$ for negative dependence. 


\section{References}

Bao, Y., T.-H. Lee, and B. Saltoğlu (2005), "Comparing Density Forecast Models", UCR.

Breymann, W., A. Dias, P. Embrechts (2003), "Dependence Structures for Multivariate High-Frequency Data in Finance", Quantitative Finance 3, 1-16.

Chen, X. and Y. Fan (2005), "Estimation and Model Selection of Semiparametric CopulaBased Multivariate Dynamic Models under Copula Misspecification", Journal of Econometrics, forthcoming.

Diebold, F.X., T.A. Gunther, and A. S. Tay (1998), "Evaluating Density Forecasts with Applications to Financial Risk Management", International Economic Review 39, 863883.

Embrechts, P., A. McNeil, and D. Straumann (1999), "Correlation: Pitfalls and Alternatives", ETH Zentrum.

Engle, R.F. (2002), "Dynamic Conditional Correlation: A Simple Class of Multivariate Generalized Autoregressive Conditional Heteroskedasticity Models", Journal of Business and Economic Statistics 20, 339-350.

Engle, R.F. and K.F. Kroner (1995), "Multivariate Simultaneous Generalized ARCH", Econometric Theory 11, 122-50.

Fama, E. and K. French (1993), Common Risk Factors in the Returns on Stocks and Bonds, Journal of Financial Economics 33, 3-56.

Granger, C.W.J. and M.H. Pesaran (2000), "Economic and Statistical Measures of Forecast Accuracy," Journal of Forecasting 19, 537-560.

Hansen, P.R. (2001), "An Unbiased and Powerful for Superior Predictive Ability", Stanford.

Hoeffding, W. (1940), Masstabinvariante Korrelationstheorie, Schriten des Matematischen Instituts und des Instituts für angewandte Mathematik der Universität Berlin, 5, Heft 3, 179-233. [Reprinted as Scale-invariant correlation theory in The Collected Works of Wassily Hoeffding, N.I. fisher and P.K. Sen editors, Springer-Verlag, New York, 57-107].

Hu, L. (2003), "Dependence Patterns across Financial Markets: a Mixed Copula Approach", OSU.

Lehmann, E.L. (1966), "Some Concepts of Dependence", Annals of Mathematical Statistics $37,1137-1153$.

Longin, F. and B. Solnik (2001), "Extreme Correlation of International Equity Market", Journal of Finance 56, 649-679.

Mashal, R. and A. Zeevi (2002), "Beyond Correlation: Extreme Co-movements Between financial Assets", Columbia University.

Nelsen, R.B. (1999), An Introduction to Copulas, Springer-Verlag, New York.

Perkins, P. and T. Lane (2003), "Monte-Carlo Simulation in MATLAB Using Copulas", MATLAB News \& Notes, November 2003. 
Richardson, M.P. and T. Smith (1993), "A Test of Multivariate Normality of Stock Returns", Journal of Business 66, 295-321.

Shea, G.A. (1983), "Hoeffding's Lemma", Encyclopedia of Statistical Sciences, Vol. 3, S. Kotz and N.L. Johnson (editors), John Wiley \& Sons, New York, 648-649.

Sklar, A. (1959), "Fonctions de répartition à n dimensions et leurs marges", Publications de Institut Statistique de Universite de Paris 8, 229-231.

Tse, Y.K. and A.K. Tsui (2002), "A Multivariate Generalized Autoregressive Conditional Heteroscedasticity Model with Time-Varying Correlations", Journal of Business and Economic Statistics 20, 351-362.

Vuong, Q. (1989), "Likelihood Ratio Tests for Model Selection and Non-nested Hypotheses", Econometrica 57, 307-333.

White, H. (1994), Estimation, Inference, and Specification Analysis. Cambridge University Press.

White, H. (2000), "A Reality Check for Data Snooping", Econometrica 68, 1097-1126. 
Table 1. Monte Carlo

\begin{tabular}{|c|c|c|c|c|c|c|c|c|c|}
\hline \multicolumn{10}{|c|}{ Panel A. F-DCC with Frank Copula } \\
\hline True $\alpha$ & 1.0000 & 2.0000 & 3.0000 & 4.0000 & 5.0000 & 6.0000 & 7.0000 & 8.0000 & 9.0000 \\
\hline$\hat{\alpha}$ from F-DCC & $\begin{array}{c}0.8608 \\
(0.9998)\end{array}$ & $\begin{array}{c}1.7702 \\
(0.9738)\end{array}$ & $\begin{array}{c}2.7835 \\
(1.0421)\end{array}$ & $\begin{array}{c}3.8464 \\
(1.0077)\end{array}$ & $\begin{array}{c}4.7327 \\
(1.0488)\end{array}$ & $\begin{array}{c}5.8254 \\
(1.1026) \\
\end{array}$ & $\begin{array}{c}6.8470 \\
(1.2313)\end{array}$ & $\begin{array}{c}7.7969 \\
(1.1130)\end{array}$ & $\begin{array}{r}8.8220 \\
(1.1839)\end{array}$ \\
\hline True $\sigma_{12}$ & 0.1570 & 0.3010 & 0.4260 & 0.5280 & 0.6092 & 0.6727 & 0.7222 & 0.7611 & 0.7919 \\
\hline$\hat{\sigma}_{12}$ from F-DCC & $\begin{array}{c}0.1313 \\
(0.1480) \\
\end{array}$ & $\begin{array}{c}0.2618 \\
(0.1355) \\
\end{array}$ & $\begin{array}{r}0.3894 \\
(0.1269) \\
\end{array}$ & $\begin{array}{c}0.5032 \\
(0.1024) \\
\end{array}$ & $\begin{array}{r}0.5789 \\
(0.0933) \\
\end{array}$ & $\begin{array}{c}0.6536 \\
(0.0732) \\
\end{array}$ & $\begin{array}{r}0.7066 \\
(0.0651) \\
\end{array}$ & $\begin{array}{c}0.7480 \\
(0.0510) \\
\end{array}$ & $\begin{array}{r}0.7823 \\
(0.0373) \\
\end{array}$ \\
\hline $\begin{array}{l}\log \mathrm{L} \text { of I-DCC } \\
\log \mathrm{L} \text { of F-DCC }\end{array}$ & $\begin{array}{l}-1473.6 \\
(78.694) \\
-1473.8 \\
(78.612) \\
\end{array}$ & $\begin{array}{l}-1472.6 \\
(78.387) \\
-1471.3 \\
(78.480) \\
\end{array}$ & $\begin{array}{l}-1470.7 \\
(78.732) \\
-1467.3 \\
(78.678) \\
\end{array}$ & $\begin{array}{l}-1468.3 \\
(79.545) \\
-1462.0 \\
(79.251) \\
\end{array}$ & $\begin{array}{l}-1465.4 \\
(80.629) \\
-1455.5 \\
(80.271) \\
\end{array}$ & $\begin{array}{l}-1462.1 \\
(81.848) \\
-1448.3 \\
(81.078) \\
\end{array}$ & $\begin{array}{l}-1458.6 \\
(83.127) \\
-1440.5 \\
(82.365) \\
\end{array}$ & $\begin{array}{l}-1454.9 \\
(84.469) \\
-1432.1 \\
(83.120) \\
\end{array}$ & $\begin{array}{l}-1451.1 \\
(85.779) \\
-1423.4 \\
(84.171) \\
\end{array}$ \\
\hline \multicolumn{10}{|c|}{ Panel B. F-VC with Frank Copula } \\
\hline True $\alpha$ & 1.0000 & 2.0000 & 3.0000 & 4.0000 & 5.0000 & 6.0000 & 7.0000 & 8.0000 & 9.0000 \\
\hline$\hat{\alpha}$ from F-VC & $\begin{array}{c}0.9303 \\
(1.0516) \\
\end{array}$ & $\begin{array}{c}1.9277 \\
(1.1568) \\
\end{array}$ & $\begin{array}{c}2.9154 \\
(1.0759) \\
\end{array}$ & $\begin{array}{c}3.9066 \\
(1.1451) \\
\end{array}$ & $\begin{array}{r}4.9443 \\
(1.2550) \\
\end{array}$ & $\begin{array}{c}5.9528 \\
(1.3379) \\
\end{array}$ & $\begin{array}{c}6.9228 \\
(1.3681) \\
\end{array}$ & $\begin{array}{c}7.9088 \\
(1.3709) \\
\end{array}$ & $\begin{array}{r}8.8879 \\
(1.3796) \\
\end{array}$ \\
\hline True $\sigma_{12}$ & 0.1570 & 0.3010 & 0.4260 & 0.5280 & 0.6092 & 0.6727 & 0.7222 & 0.7611 & 0.7919 \\
\hline$\hat{\sigma}_{12}$ from F-VC & $\begin{array}{c}0.1402 \\
(0.1539)\end{array}$ & $\begin{array}{c}0.2791 \\
(0.1524)\end{array}$ & $\begin{array}{c}0.4038 \\
(0.1235)\end{array}$ & $\begin{array}{c}0.5061 \\
(0.1083)\end{array}$ & $\begin{array}{c}0.5914 \\
(0.0972)\end{array}$ & $\begin{array}{c}0.6576 \\
(0.0811)\end{array}$ & $\begin{array}{c}0.7087 \\
(0.0645)\end{array}$ & $\begin{array}{c}0.7502 \\
(0.0507)\end{array}$ & $\begin{array}{r}0.7828 \\
(0.0419) \\
\end{array}$ \\
\hline $\begin{array}{l}\log \mathrm{L} \text { of } \mathrm{I}-\mathrm{VC} \\
\log \mathrm{L} \text { of } \mathrm{F}-\mathrm{VC}\end{array}$ & $\begin{array}{l}-1433.7 \\
(76.268) \\
-1433.9 \\
(76.430) \\
\end{array}$ & $\begin{array}{l}-1429.2 \\
(76.929) \\
-1427.9 \\
(77.020) \\
\end{array}$ & $\begin{array}{l}-1424.4 \\
(78.305) \\
-1420.6 \\
(78.365) \\
\end{array}$ & $\begin{array}{l}-1418.9 \\
(79.883) \\
-1412.2 \\
(79.715) \\
\end{array}$ & $\begin{array}{l}-1413.1 \\
(81.186) \\
-1402.9 \\
(80.699) \\
\end{array}$ & $\begin{array}{l}-1407.3 \\
(82.406) \\
-1392.9 \\
(81.616) \\
\end{array}$ & $\begin{array}{c}-1401.6 \\
(83.959) \\
-1382.9 \\
(82.882) \\
\end{array}$ & $\begin{array}{l}-1395.7 \\
(85.133) \\
-1372.1 \\
(83.853) \\
\end{array}$ & $\begin{array}{l}-1390.2 \\
(86.708) \\
-1361.5 \\
(84.971) \\
\end{array}$ \\
\hline \multicolumn{10}{|c|}{ Panel C. F-BEKK with Frank Copula } \\
\hline True $\alpha$ & 1.0000 & 2.0000 & 3.0000 & 4.0000 & 5.0000 & 6.0000 & 7.0000 & 8.0000 & 9.0000 \\
\hline$\hat{\alpha}$ from F-BEKK & $\begin{array}{c}0.9039 \\
(0.9911) \\
\end{array}$ & $\begin{array}{c}1.8796 \\
(1.0748)\end{array}$ & $\begin{array}{c}2.7570 \\
(1.0731)\end{array}$ & $\begin{array}{c}3.6863 \\
(0.9843) \\
\end{array}$ & $\begin{array}{c}4.6623 \\
(1.0090) \\
\end{array}$ & $\begin{array}{c}5.7183 \\
(1.1717) \\
\end{array}$ & $\begin{array}{c}6.6872 \\
(1.2013) \\
\end{array}$ & $\begin{array}{c}7.6180 \\
(1.2739) \\
\end{array}$ & $\begin{array}{c}8.6128 \\
(1.5426) \\
\end{array}$ \\
\hline True $\sigma_{12}$ & 0.1570 & 0.3010 & 0.4260 & 0.5280 & 0.6092 & 0.6727 & 0.7222 & 0.7611 & 0.7919 \\
\hline$\hat{\sigma}_{12}$ from F-BEKK & $\begin{array}{r}0.1379 \\
(0.1465) \\
\end{array}$ & $\begin{array}{c}0.2749 \\
(0.1444) \\
\end{array}$ & $\begin{array}{r}0.3855 \\
(0.1286) \\
\end{array}$ & $\begin{array}{c}0.4880 \\
(0.1012) \\
\end{array}$ & $\begin{array}{c}0.5742 \\
(0.0861) \\
\end{array}$ & $\begin{array}{c}0.6461 \\
(0.0779) \\
\end{array}$ & $\begin{array}{c}0.6994 \\
(0.0642) \\
\end{array}$ & $\begin{array}{r}0.7394 \\
(0.0583) \\
\end{array}$ & $\begin{array}{r}0.7733 \\
(0.0481) \\
\end{array}$ \\
\hline $\operatorname{logL}$ of I-BEKK & $\begin{array}{l}-800.31 \\
(53.775)\end{array}$ & $\begin{array}{l}-798.59 \\
(53.807)\end{array}$ & $\begin{array}{l}-796.14 \\
(54.418)\end{array}$ & $\begin{array}{l}-793.09 \\
(55.225)\end{array}$ & $\begin{array}{l}-789.54 \\
(56.382)\end{array}$ & $\begin{array}{l}-785.94 \\
(57.232)\end{array}$ & $\begin{array}{l}-781.91 \\
(58.418)\end{array}$ & $\begin{array}{l}-777.99 \\
(59.535)\end{array}$ & $\begin{array}{l}-773.94 \\
(60.784)\end{array}$ \\
\hline $\log \mathrm{L}$ of F-BEKK & $\begin{array}{l}-799.37 \\
(53.752) \\
\end{array}$ & $\begin{array}{l}-796.28 \\
(53.880) \\
\end{array}$ & $\begin{array}{l}-791.49 \\
(54.412) \\
\end{array}$ & $\begin{array}{c}-785.40 \\
(55.101) \\
\end{array}$ & $\begin{array}{r}-778.39 \\
(55.930) \\
\end{array}$ & $\begin{array}{r}-770.58 \\
(56.802) \\
\end{array}$ & $\begin{array}{l}-762.20 \\
(57.708) \\
\end{array}$ & $\begin{array}{l}-753.25 \\
(58.573) \\
\end{array}$ & $\begin{array}{l}-743.99 \\
(59.210) \\
\end{array}$ \\
\hline
\end{tabular}

Notes: The sample size is 500 and the number of Monte Carlo replications is 200. $\hat{\alpha}$ denotes the Monte Carlo mean of the estimated shape parameter $\alpha$. $\sigma_{12}$ is the off-diagonal element of $\Sigma(\alpha)$ corresponding to true value of $\alpha$. $\hat{\sigma}_{12}$ denotes the Monte Carlo mean of the off-diagonal element of $\boldsymbol{\Sigma}(\hat{\alpha})$. $\log \mathrm{L}$ denotes the Monte Carlo mean of the log-likelihood. The numbers in parentheses are the Monte Carlo standard deviations for $\hat{\alpha}, \hat{\sigma}_{12}$, and logL. F-DCC denotes the Frank copula based DCC, and I-DCC denotes the independent copula based DCC. Similar notation is used for F-VC, I-VC, FBEKK, and I-BEKK. The Monte Carlo results are reported only with Frank copula for space, but those with Clayton copula and Gumbel copula are available upon request. All three copula functions deliver more or less the same results. 
Table 2. In-Sample Estimation

NASDAQ and Dow Jones

Panel A1. DCC Family for NASDAQ and Dow Jones

\begin{tabular}{l|c|cccccc}
\hline & $\mathrm{I}$ & $\mathrm{G}$ & $\mathrm{GS}$ & $\mathrm{JC}$ & $\mathrm{C}$ & $\mathrm{CS}$ & $\mathrm{F}$ \\
\hline$\alpha_{1}$ & & 1.0233 & 1.0542 & 1.0323 & 0.1209 & 0.0162 & -1.3484 \\
& & $(0.7905)$ & $(0.0954)$ & $\begin{array}{c}(2.3055) \\
(0.0416)\end{array}$ & $(0.2516)$ & $(0.8253)$ \\
$\alpha_{2}$ & & & & 0.2030 & & & \\
& & & & $(2.8137)$ & & & -0.2088 \\
$\sigma_{12}$ & & 0.0375 & 0.0839 & 0.1747 & 0.0924 & 0.0130 & -4098.3 \\
\hline $\log \mathrm{AIC}$ & -4106.5 & -4101.0 & -4084.0 & $\mathbf{- 4 0 8 3 . 2}$ & -4092.8 & -4100.1 & 6.5403 \\
SIC & 6.5518 & 6.5446 & $\mathbf{6 . 5 1 7 5}$ & 6.5178 & 6.5315 & 6.5432 & 6.5482 \\
\hline
\end{tabular}

Panel A2. VC Family for NASDAQ and Dow Jones

\begin{tabular}{|c|c|c|c|c|c|c|c|}
\hline & $\mathrm{I}$ & $\mathrm{G}$ & GS & $\mathrm{JC}$ & $\mathrm{C}$ & CS & $\mathrm{F}$ \\
\hline $\begin{array}{l}\alpha_{1} \\
\alpha_{2}\end{array}$ & & $\begin{array}{c}1.7208 \\
(0.6641)\end{array}$ & $\begin{array}{c}1.0531 \\
(0.0392)\end{array}$ & $\begin{array}{c}1.1842 \\
(0.2927) \\
0.3498 \\
(0.2429)\end{array}$ & $\begin{array}{c}0.1475 \\
(0.0722)\end{array}$ & $\begin{array}{c}0.0071 \\
(0.0977)\end{array}$ & $\begin{array}{c}-1.7023 \\
(0.8524)\end{array}$ \\
\hline$\sigma_{12}$ & & 0.6049 & 0.0823 & 0.3502 & 0.1112 & 0.0057 & -0.2599 \\
\hline $\begin{array}{l}\log L \\
\text { AIC } \\
\text { SIC } \\
\end{array}$ & $\begin{array}{c}-4122.0 \\
6.5764 \\
6.5834 \\
\end{array}$ & $\begin{array}{c}-4134.1 \\
6.5973 \\
6.6052 \\
\end{array}$ & $\begin{array}{c}-4099.3 \\
6.5419 \\
6.5498 \\
\end{array}$ & $\begin{array}{c}-4096.9 \\
6.5396 \\
6.5484 \\
\end{array}$ & $\begin{array}{c}-4110.6 \\
6.5599 \\
6.5677 \\
\end{array}$ & $\begin{array}{c}-4116.8 \\
6.5697 \\
6.5776 \\
\end{array}$ & $\begin{array}{c}-4111.3 \\
6.5610 \\
6.5689 \\
\end{array}$ \\
\hline
\end{tabular}

Panel A3. BEKK Family for NASDAQ and Dow Jones

\begin{tabular}{l|c|cccccc}
\hline & $\mathrm{I}$ & $\mathrm{G}$ & $\mathrm{GS}$ & $\mathrm{JC}$ & $\mathrm{C}$ & $\mathrm{CS}$ & $\mathrm{F}$ \\
\hline$\alpha_{1}$ & & 1.1046 & 1.1030 & 1.3153 & 0.1794 & 0.1629 & -4.9751 \\
& & $(0.2068)$ & $(1.0270)$ & $\begin{array}{c}(0.4598) \\
(0.2477\end{array}$ & $(0.0764)$ & $(0.3231)$ & $(1.1179)$ \\
$\alpha_{2}$ & & & & $(0.1950)$ & & & \\
$\sigma_{12}$ & & & & & & & \\
\hline $\operatorname{logL}$ & -4185.9 & -4313.5 & $\mathbf{- 4 0 9 8 . 1}$ & -4105.1 & -4108.2 & -4131.0 & -4126.6 \\
$\mathrm{AIC}$ & 6.6686 & 6.8734 & $\mathbf{6 . 5 3 0 4}$ & 6.5432 & 6.5465 & 6.5828 & 6.5758 \\
$\mathrm{SIC}$ & 6.6704 & 6.8760 & $\mathbf{6 . 5 3 3 0}$ & 6.5467 & 6.5491 & 6.5854 & 6.5784 \\
\hline
\end{tabular}

Notes: Daily changes in log stock indexes for NASDAQ and Dow Jones from January 2, 1997 to December 31, $2001(T=1257)$ are used. Estimated are seven C-MGARCH models with Independent copula (I), Gumbel copula (G), Gumbel Survival copula (GS), Joe-Clayton copula (JC), Clayton copula (C), Clayton Survival copula (CS), and Frank copula (F). For space, only copula shape parameter estimates and their robust standard errors are reported. The parameter estimates are from the one-step QMLE. The numbers in parentheses are the standard errors calculated from the robust QMLE covariance matrix of the parameters. $\alpha_{1}$ and $\alpha_{2}$ are the copula shape parameters. The Joe-Clayton copula function has two shape parameters while each of the other copula functions has only one shape parameter. $\sigma_{12}$ is the off-diagonal element of $\Sigma$. The largest $\log$-likelihood ( $\log \mathrm{L})$ and the smallest AIC and SIC for each family are in bold font to indicate the best C-MARCH model of each family. 
Table 2. In-Sample Estimation (Continued)

French Franc and Deutschemark

Panel B1. DCC Family for French Franc and Deutschemark

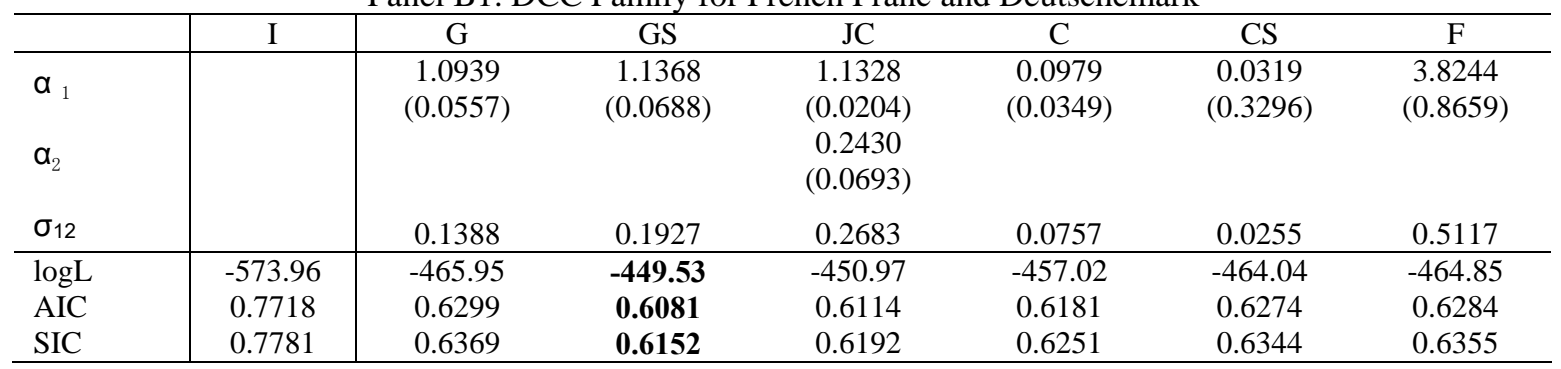

Panel B2. VC Family for French Franc and Deutschemark

\begin{tabular}{|c|c|c|c|c|c|c|c|}
\hline & I & G & GS & $\mathrm{JC}$ & C & CS & $\mathrm{F}$ \\
\hline $\begin{array}{l}\alpha_{1} \\
\alpha_{2}\end{array}$ & & $\begin{array}{c}1.0469 \\
(0.1188)\end{array}$ & $\begin{array}{c}1.1594 \\
(0.0867)\end{array}$ & $\begin{array}{c}1.1071 \\
(0.1284) \\
0.2077 \\
(0.1327)\end{array}$ & $\begin{array}{c}0.1928 \\
(1.2554)\end{array}$ & $\begin{array}{c}0.0088 \\
(1.8694)\end{array}$ & $\begin{array}{c}2.6077 \\
(0.8974)\end{array}$ \\
\hline$\sigma_{12}$ & & 0.0733 & 0.2191 & 0.2320 & 0.1421 & 0.0071 & 0.3796 \\
\hline $\log L$ & -591.62 & -494.19 & -479.05 & -479.98 & -490.00 & -496.65 & -489.84 \\
\hline AIC & 0.7953 & 0.6674 & 0.6473 & 0.6498 & 0.6618 & 0.6706 & 0.6616 \\
\hline SIC & 0.8015 & 0.6744 & 0.6543 & 0.6577 & 0.6688 & 0.6777 & 0.6686 \\
\hline
\end{tabular}

Panel B3. BEKK Family for French Franc and Deutschemark

\begin{tabular}{|c|c|c|c|c|c|c|c|}
\hline & $\mathrm{I}$ & $G$ & GS & $\mathrm{JC}$ & $\mathrm{C}$ & $\mathrm{CS}$ & $\mathrm{F}$ \\
\hline $\begin{array}{l}\alpha_{1} \\
\alpha_{2}\end{array}$ & & $\begin{array}{c}1.0323 \\
(0.0502)\end{array}$ & $\begin{array}{c}1.0864 \\
(0.0473)\end{array}$ & $\begin{array}{c}1.1005 \\
(0.1369) \\
0.1761 \\
(0.1195)\end{array}$ & $\begin{array}{c}0.1350 \\
(0.0442)\end{array}$ & $\begin{array}{c}0.0047 \\
(0.0360)\end{array}$ & $\begin{array}{c}1.8904 \\
(1.0887)\end{array}$ \\
\hline$\sigma_{12}$ & & 0.0514 & 0.1289 & 0.2089 & 0.1025 & 0.0038 & 0.2862 \\
\hline $\log L$ & -548.22 & -459.44 & -449.07 & -447.62 & -456.24 & -461.56 & -457.27 \\
\hline AIC & 0.7297 & 0.6133 & 0.5996 & 0.5990 & 0.6091 & 0.6161 & 0.6104 \\
\hline SIC & 0.7313 & 0.6157 & 0.6019 & 0.6021 & 0.6114 & 0.6185 & 0.6128 \\
\hline
\end{tabular}

Notes: Daily changes in log exchange rates for French Franc and Deutschemark from January 4, 1993 to December 31, $1998(T=1508)$ are used. Also see Notes for Panel A. 
Table 2. In-Sample Estimation (Continued)

Japanese Yen and Deutschemark

Panel C1. DCC Family for Japanese Yen and Deutschemark

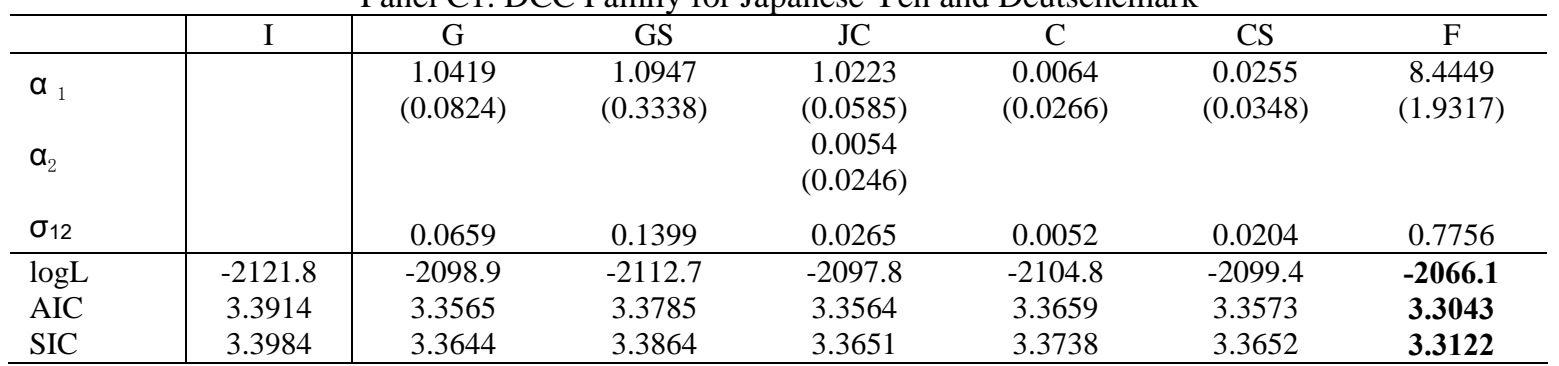

Panel C2. VC Family for Japanese Yen and Deutschemark

\begin{tabular}{|c|c|c|c|c|c|c|c|}
\hline & $\mathrm{I}$ & $\mathrm{G}$ & GS & $\mathrm{JC}$ & $\mathrm{C}$ & CS & $\mathrm{F}$ \\
\hline $\begin{array}{l}\alpha_{1} \\
\alpha_{2}\end{array}$ & & $\begin{array}{c}1.0579 \\
(0.1084)\end{array}$ & $\begin{array}{c}1.0229 \\
(0.0969)\end{array}$ & $\begin{array}{c}1.0164 \\
(0.0831) \\
0.0084 \\
(0.0451)\end{array}$ & $\begin{array}{c}0.0092 \\
(0.0266)\end{array}$ & $\begin{array}{c}0.0195 \\
(0.0359)\end{array}$ & $\begin{array}{c}8.3790 \\
(1.7274)\end{array}$ \\
\hline$\sigma_{12}$ & & 0.0893 & 0.0368 & 0.0231 & 0.0074 & 0.0157 & 0.7736 \\
\hline $\log L$ & -2119.3 & -2096.1 & -2098.4 & -2096.9 & -2096.7 & -2096.8 & -2066.7 \\
\hline AIC & 3.3874 & 3.3521 & 3.3557 & 3.3549 & 3.3530 & 3.3532 & 3.3053 \\
\hline SIC & 3.3944 & 3.3599 & 3.3636 & 3.3637 & 3.3609 & 3.3611 & 3.3131 \\
\hline
\end{tabular}

Panel C3. BEKK Family for Japanese Yen and Deutschemark

\begin{tabular}{|c|c|c|c|c|c|c|c|}
\hline & I & G & GS & $\mathrm{JC}$ & $\mathrm{C}$ & CS & $\mathrm{F}$ \\
\hline $\begin{array}{l}\alpha_{1} \\
\alpha_{2}\end{array}$ & & $\begin{array}{c}1.0498 \\
(0.0932)\end{array}$ & $\begin{array}{c}1.0099 \\
(0.0690)\end{array}$ & $\begin{array}{c}1.0524 \\
(0.0903) \\
0.0233 \\
(0.0343)\end{array}$ & $\begin{array}{c}0.0050 \\
(0.0255)\end{array}$ & $\begin{array}{c}0.0331 \\
(0.0409)\end{array}$ & $\begin{array}{c}6.9826 \\
(1.5707)\end{array}$ \\
\hline$\sigma_{12}$ & & 0.0775 & 0.0162 & 0.0684 & 0.0040 & 0.0264 & 0.7215 \\
\hline $\log L$ & -2128.9 & -2104.8 & -2106.5 & -2106.2 & -2105.8 & -2106.9 & -2078.6 \\
\hline AIC & 3.3932 & 3.3564 & 3.3591 & 3.3602 & 3.3580 & 3.3597 & 3.3146 \\
\hline SIC & 3.3949 & 3.3590 & 3.3617 & 3.3637 & 3.3606 & 3.3623 & 3.3173 \\
\hline
\end{tabular}

Notes: Daily changes in log exchange rates for Japanese Yen and the Deutschemark from January 4, 1993 to December 31, 1997 ( $T=1257$ ) are used. Also see Notes for Panel A. 
Table 3. Out-of-Sample Predictive Ability

\begin{tabular}{l|ccc|ccc|ccc}
\hline & \multicolumn{3}{|c|}{$\begin{array}{c}\text { NASDAQ and } \\
\text { Dow Jones }\end{array}$} & \multicolumn{3}{c|}{$\begin{array}{c}\text { French Franc and } \\
\text { Deutschemark }\end{array}$} & \multicolumn{3}{c}{$\begin{array}{c}\text { Japanese Yen and } \\
\text { Deutschemark }\end{array}$} \\
\hline Copula & DCC & VC & BEKK & DCC & VC & BEKK & DCC & VC & BEKK \\
\hline I & -3.5904 & -3.5957 & -3.5941 & 0.0604 & -0.0731 & 0.0725 & -1.9418 & -1.9452 & -1.9356 \\
G & -3.5663 & -3.5770 & -3.5757 & 0.1029 & -0.0330 & 0.0893 & -1.9425 & -1.9448 & -1.9322 \\
GS & $\mathbf{- 3 . 5 6 0 3}$ & -3.5712 & -3.5721 & 0.1022 & -0.0068 & 0.0889 & -1.9412 & -1.9432 & -1.9388 \\
JC & -3.5661 & $\mathbf{- 3 . 5 6 7 1}$ & $\mathbf{- 3 . 5 6 7 7}$ & $\mathbf{0 . 1 1 1 4}$ & $\mathbf{- 0 . 0 0 1 8}$ & $\mathbf{0 . 1 0 4 8}$ & -1.9403 & -1.9393 & -1.9382 \\
C & -3.5737 & -3.5797 & -3.5700 & 0.0927 & -0.0309 & 0.0895 & -1.9455 & -1.9458 & -1.9391 \\
CS & -3.5683 & -3.5795 & -3.5704 & 0.0956 & -0.0431 & 0.0848 & -1.9434 & -1.9451 & -1.9338 \\
F & -3.5630 & -3.5743 & -3.5696 & 0.1091 & -0.0344 & 0.0898 & $\mathbf{- 1 . 9 1 0 6}$ & $\mathbf{- 1 . 9 0 9 3}$ & $\mathbf{- 1 . 9 0 6 6}$ \\
\hline White & 0.087 & 0.095 & 0.195 & 0.002 & 0.000 & 0.008 & 0.184 & 0.144 & 0.181 \\
Hansen & 0.087 & 0.095 & 0.195 & 0.002 & 0.000 & 0.008 & 0.184 & 0.144 & 0.181 \\
\hline
\end{tabular}

Notes: The out-of-sample average of predictive likelihood is reported. The best model for each family with the largest value of the out-of-sample average predictive likelihood is in bold font. For each of DCC, VC, BEKK families, seven C-MGARCH models are recursively estimated to generate $P$ one-step density forecasts over the out-of-sample validation samples. We use the rolling window of size $R$ to train the forecasting models with $R=1009,1009,1007$ for the three data sets. The seven copulas used are Independent copula (denoted as I), Gumbel copula (G), Gumbel Survival copula (GS), Joe-Clayton copula (JC), Clayton copula (C), Clayton Survival copula (CS), and Frank copula (F). The range of the out-ofsample is from January 4, 2001 to December 31, $2001(P=248)$ for NASDAQ and Dow-Jones, from January 7, 1997 to December 31, $1998(P=500)$ for French Franc and Deutschemark, and from January 5, 1997 to December 31, $1997(P=250)$ for Japanese Yen and Deutschemark. For reality check, we use 1000 bootstrap samples with the mean block size of the "stationary bootstrap" equal to 5 days (a week), i.e., with the stationary bootstrap parameter $q=0.2$. The benchmark model in each family is the independent copula model. "White" refers to the bootstrap reality check p-value of White (2000) and "Hansen" refers to the bootstrap reality check p-value of Hansen (2001). As discussed in Hansen (2001), White's reality check pvalue may be considered as an upper bound of the true p-value. Hansen (2001) considers a modified reality check test to improve the size and the power of the test. White's p-value and Hansen's p-value may be the same, as is the case in this table. Nevertheless, we report both. 